\title{
Imaging of dynamic heterogeneities in multiple-scattering media
}

\author{
M. Heckmeier \\ Institut Charles Sadron, Centre de Recherches sur les Macromolecules-Ecole d'Applications des Hauts Polymeres, \\ 6 rue Boussingault, F-67083 Strasbourg Cedex, France \\ S. E. Skipetrov* \\ Laboratoire d'Expérimentation Numérique, Université Joseph Fourier, Maison des Magistères-Centre National \\ de la Recherche Scientifique, B.P. 166, F-38042 Grenoble Cedex, France
}

\section{G. Maret}

Institut Charles Sadron, Centre de Recherches sur les Macromolecules-Ecole d'Applications des Hauts Polymeres, 6 rue Boussingault, F-67083 Strasbourg Cedex, France

\author{
R. Maynard \\ Laboratoire d'Expérimentation Numérique, Université Joseph Fourier, Maison des Magistères-Centre National \\ de la Recherche Scientifique, B.P. 166, F-38042 Grenoble Cedex, France
}

Received April 1, 1996; revised manuscript received July 8, 1996; accepted July 10, 1996

\begin{abstract}
A new method of visualizing objects with distinct internal dynamics of the constituent scattering particles embedded in a liquid multiple-scattering medium is presented. We report dynamic multiple-light-scattering experiments and a theoretical model, based on diffusing photon-density waves for concentrated colloidal suspensions in Brownian motion, as a background medium into which is inserted a capillary containing (i) the same suspension under flow, or (ii) suspensions of different particle sizes in Brownian motion. These model objects, with purely dynamic but no static scattering contrast, can be visualized by space-resolved measurements of the time autocorrelation function $g_{2}(\tau)$ of the scattered light intensity at the sample surface. Maximum contrast occurs at a parameter-dependent finite correlation time $\tau$. The physical origin of this effect is outlined. Our data are in excellent quantitative agreement with the model, with no adjustable parameter. (C) 1997 Optical Society of America. [S0740-3232(97)00701-1]
\end{abstract}

\section{INTRODUCTION}

Dynamic fluctuations of multiple-scattered light from turbid but macroscopically homogeneous liquid suspensions have been widely investigated in recent years, both theoretically and experimentally. The time autocorrelation function $g_{2}(\tau)$ of the scattered light intensity provides unambiguous quantitative information about various types of motion of the scattering particles, as may be illustrated by research on Brownian motion, ${ }^{1}$ short-time ballistic motion, ${ }^{2}$ shear flow, ${ }^{3,4}$ acoustic modulation, ${ }^{5}$ or structural rearrangement of foams. ${ }^{6}$ In fact, the different motions generate rather characteristic time dependences of $g_{2}(\tau)$.

At the same time, substantial interest has developed in the effort to image or to locate objects that are buried more deeply than a few scattering mean free paths of light inside a multiple-scattering medium. Many possible applications, particularly in medical sciences, are based on the fact that an absorbing or transmitting object modifies the spatial distribution of the diffusing photon density. As a consequence, the light intensity emerging from the sample surface contains some information about the object's position and size. ${ }^{7,8}$

Combining the two above-mentioned features makes it possible to localize or to image objects consisting of scattering particles undergoing a motion that differs from the particle's motion outside the object. ${ }^{9,10}$ Boas et al. ${ }^{9}$ have visualized a spherical inclusion filled with a colloidal suspension in Brownian motion surrounded by a solid multiple-scattering slab, with the contrast being in the particle dynamics and in the static scattering properties. Heckmeier and Maret ${ }^{10}$ have visualized shear flow of a colloidal suspension confined in a capillary embedded in the same suspension, thus exploiting dynamic contrast alone. In these experiments the use of a backscattering geometry is very convenient and should be more appropriate for most practical applications.

In this paper we extend the measurements reported in Ref. 10 to dynamic contrast that is due to differential Brownian motion (but no flow), using different-size particles inside and outside the capillary. In addition, we compare the data with a diffusion theory for $g_{2}(\tau)$, which is developed for two distinct sample geometries: an infinite slab of thickness $d$ and a cylindrical capillary of radius $a$, respectively, embedded in a semi-infinite slab. The parameters are chosen such that there is no static scattering contrast between the object and its environ- 
ment. Our theory quantitatively accounts for all the features of our data on flow and on differential Brownian motion. The general case of combined static and dynamic contrast is not considered here, since its theory is more involved.

\section{THEORY}

It can be shown ${ }^{9}$ that in a highly multiple-scattering regime $l^{*} \ll L$, where $l^{*}$ is the transport mean free path for elastic scattering and $L$ is a characteristic dimension of the sample, the nonnormalized temporal depolarized electric-field autocorrelation function at the position $\mathbf{r}$ within a turbid medium $G_{1}(\mathbf{r}, \tau)=\left\langle E(\mathbf{r}, t) E^{*}(\mathbf{r}, t\right.$ $+\tau)\rangle$ is well approximated by the solution of the steadystate diffusion equation. ${ }^{9,11}$ In the case of negligible light absorption, this equation can be written as

$$
\left[\nabla^{2}-k^{2}(\tau)\right] G_{1}(\mathbf{r}, \tau)=-\frac{S(\mathbf{r})}{D_{p}},
$$

where $k^{2}(\tau)$ describes the attenuation of temporal correlation with time, which is discussed below; $D_{p}=v l^{*} / 3(v$ denotes the speed of light in the medium) is the photon diffusion coefficient; and $S(\mathbf{r})$ is a light-source distribution. In the case of pure Brownian motion of scatterers $k^{2}(\tau)=3 \tau /\left(2 \tau_{0} l^{* 2}\right)$, where $\tau_{0}=\left(4 k_{0}^{2} D_{B}\right)^{-1}$, with $D_{B}$ being the particle diffusion coefficient and $k_{0}$ the wave number of the light in the medium. This type of diffusion equation was initially derived from the transport theory of light in a macroscopically homogeneous medium ${ }^{12}$ for a correlation function measured far from its boundaries and far from the light sources. It was shown, however, that Eq. (1) is also valid even when these conditions are not strictly fulfilled. Thus, for instance, Eq. (1) gives correct results not only for the correlation function of light transmitted or reflected from a slab filled with a turbid medium $^{12,13}$ but also for turbid media with large-scale spatially varying dynamics. ${ }^{9}$ For that case an expression for $G_{1}(\mathbf{r}, \tau)$ that considers the Brownian motion of scatterers as a source of wave dephasing has been derived. Here we generalize this description to the situation of Poiseuille flow of scatterers, which is simply done by changing of the $\tau$ dependence of $k^{2}(\tau)$.

We consider a semi-infinite medium that occupies the half-space $x>0$ in which is embedded a hidden inclusion (the object) of volume $V_{1} \gg l^{* 3}$ limited by the surface $S_{1}$. This inclusion is characterized by different dynamics of scatterers as compared with the surrounding medium. To describe this difference we introduce an additional spatial dependence of $k^{2}$ in the form $k^{2}(\mathbf{r}, \tau)=k_{\text {in }}{ }^{2}(\tau)$ inside $V_{1}$ and $k^{2}(\mathbf{r}, \tau)=k_{\text {out }}{ }^{2}(\tau)$ elsewhere. Such an approach is valid only for sufficiently large inclusions, that is, when the diffusion approximation is applicable inside the inclusion. Since we are interested in purely dynamic heterogeneities, we assume the photon diffusion coefficient or the photon transport mean free path to be constant within the entire scattering volume, inside and outside $V_{1}$.

We are interested in the case of flow of scatterers inside either a cylindrical tube or a slab with volume $V_{1}$ in which Brownian motion also exists. If the scatterers in the surrounding medium undergo only Brownian motion, we have $k_{\text {in }}{ }^{2}(\tau)=3 \tau /\left(2 \tau_{0}{ }^{\text {in }} l^{* 2}\right)+6\left[\tau /\left(\tau_{f} l^{*}\right)\right]^{2}$ and $k_{\text {out }}{ }^{2}(\tau)=3 \tau /\left(2 \tau_{0}{ }^{\text {out }} l * 2\right)$, where $\tau_{0}^{\text {in,out }}$ are related to the particle diffusion coefficients $D_{B}$ in,out inside and outside $V_{1}$ through $\tau_{0}^{\text {in,out }}=\left(4 k_{0}{ }^{2} D_{B}{ }^{\text {in,out }}\right)^{-1}$ and $\tau_{f}$ is a characteristic time introduced by the flow.

To distinguish between perturbations of correlation arising from the presence of flow and those determined by different particle diffusion coefficients inside and outside the inclusion, we consider the two following cases: (i) that in which the flow of a fluid suspension is surrounded by exactly the same medium $\left(\tau_{f}<\infty, \tau_{0}{ }^{\text {in }}=\tau_{0}{ }^{\text {out }}\right)$, and (ii) that of the purely Brownian inhomogeneity $\left(\tau_{f} \rightarrow \infty\right.$, $\tau_{0}^{\text {in }} \neq \tau_{0}{ }^{\text {out }}$ ).

In the above formulas the term $\left(3 / 2 l^{* 2}\right)\left(\tau / \tau_{0}\right)$ describes attenuation of correlation due to the Brownian motion of scatterers, ${ }^{11,14}$ and the $\left(6 / l^{* 2}\right)\left(\tau / \tau_{f}\right)^{2}$ determines the additional loss of correlation caused by the flow. ${ }^{15}$ The characteristic time $\tau_{f}$ depends on a distribution of velocity gradients related to a particular geometry of the flow. We assume that $\tau_{f}$ is determined by the root-mean-square velocity gradient ${ }^{15} \Gamma_{1}: \tau_{f}=\sqrt{30} /\left(k_{0} l^{*} \Gamma_{1}\right) . \quad \Gamma_{1}=\Gamma / \sqrt{3}$ for planar Poiseuille flow in an infinite slice of width $d$ $=2 a\left[\mathbf{V}=\Gamma / d\left(a^{2}-x^{2}\right) \mathbf{e}_{z},-a \leqslant x \leqslant a\right]$, and $\Gamma_{1}$ $=\Gamma / \sqrt{2}$ for cylindrical Poiseuille flow in an infinite capillary of diameter $d=2 a$ [in cylindrical coordinates $\mathbf{V}$ $\left.=\Gamma / d\left(a^{2}-\rho^{2}\right) \mathbf{e}_{z}, 0 \leqslant \rho \leqslant a\right]$. In the latter case $\Gamma_{1}$ $=32 Q /\left(\sqrt{2} \pi d^{3}\right)$, where $Q$ is the flow rate. Indeed, this description means that an additional dephasing of the photons inside the region of flow depends not on the particular form of their trajectories but only on the total length of their paths inside $V_{1}$. This approximation is therefore valid only when $d \gg l^{*}$ and for long diffusion paths, which correspond to relatively short correlation times. $^{11}$

To complete the mathematical formulation of the problem, we specify the boundary conditions on the surface $S$ of the medium and on the surface $S_{1}$ of the dynamical heterogeneity ${ }^{9,16}$ :

$$
\begin{gathered}
G_{1}{ }^{\text {out }}(\mathbf{r}, \tau)-(2 / 3) l^{*}\left[\mathbf{n} \cdot \nabla G_{1}{ }^{\text {out }}(\mathbf{r}, \tau)\right]=0, \quad \mathbf{r} \in S, \\
G_{1}^{\text {in }}(\mathbf{r}, \tau)=G_{1}{ }^{\text {out }}(\mathbf{r}, \tau) \\
D_{p}{ }^{\text {in }}\left[\mathbf{n} \cdot \nabla G_{1}^{\text {in }}(\mathbf{r}, \tau)\right]=D_{p}{ }^{\text {out }}\left[\mathbf{n} \cdot \nabla G_{1}{ }^{\text {out }}(\mathbf{r}, \tau)\right] \\
\mathbf{r} \in S_{1} .
\end{gathered}
$$

Here $\mathbf{n}$ is a unit normal vector pointing inward toward the corresponding surface, and $G_{1}{ }^{i n}(\mathbf{r}, \tau), G_{1}{ }^{\text {out }}(\mathbf{r}, \tau)$, and $D_{p}{ }^{\text {in }}, D_{p}{ }^{\text {out }}$ are solutions of Eq. (1) and photon diffusion coefficients inside and outside the volume $V_{1}$, respectively. In our case of $D_{p}{ }^{\text {in }}=D_{p}{ }^{\text {out }}$ the last condition can be simplified. The first condition can be approximately replaced by a null condition for $G_{1}$ at an extrapolated boundary ${ }^{13,17} x=-x_{1}=-\Delta l^{*}$, where $\Delta$ depends sensitively on the transport of light near the sample boundary (see Ref. 4 and references therein). $\Delta_{0}=0.7104$ is found 
from the Milne theory ${ }^{14}$ for isotropic scattering and for vanishing optical index mismatch between the scattering medium and its surroundings. We can account for mismatch in the index of refraction at the surface by changing this extrapolation length, ${ }^{4,18}$ i.e., by changing $\Delta$. The boundary conditions (2) have the same form as for diffuse photon-density waves and were discussed in detail previously. ${ }^{12,16}$

To simplify our analysis, we assume a plane wave incident upon the medium surface. This results in a source term $S(\mathbf{r})=\delta\left(x-x_{0}\right)$ in Eq. (1) with $x_{0} \simeq l^{*}$. In other words, we assume that photons begin their scattering sequences at a depth $x_{0}$ of the order of a transport mean free path inside the medium. The light-collecting detector is assumed to be placed on the surface of the medium. This is experimentally realized by imaging of the sample surface on the cathode of a phototube.

In the weak multiple-scattering limit $\left(k_{0} l^{*} \gg 1\right)$ the normalized autocorrelation function of the depolarized light backscattered from a homogeneous semi-infinite sample $\left(\tau_{f} \rightarrow \infty, \tau_{0}{ }^{\text {in }}=\tau_{0}{ }^{\text {out }}\right), g_{1}(\tau)=G_{1}(\tau) / G_{1}(0)$, becomes

$$
g_{1}{ }^{0}(\tau)=\exp \left(-\gamma \sqrt{\frac{3 \tau}{2 \tau_{0}{ }^{\text {out }}}}\right)
$$

where $\gamma=1+\Delta$ is a numerical constant of order 2 and the superscript 0 of $g_{1}{ }^{0}(\tau)$ denotes the homogeneous case. This result, which was obtained previously by many investigators, is in excellent agreement with experiments. 11,16

When $V_{1}$ has the form of an infinite slice of width $d$ that is embedded at a depth $x$ inside the medium, we obtain the following equation as the solution of Eq. (1) with boundary conditions (2) in backscattering:

$$
g_{1}(\tau)=\frac{F\left(\xi_{1}\right)}{F\left(\xi_{2}\right)}
$$

where

$$
\begin{aligned}
F(\xi)= & k_{\text {in }} k_{\text {out }} \exp \left(k_{\text {out }} \xi\right)+\tanh \left(k_{\text {in }} d\right) \\
& \times\left[k_{\text {out }}^{2} \cosh \left(k_{\text {out }} \xi\right)+{k_{\text {in }}}^{2} \sinh \left(k_{\text {out }} \xi\right)\right]
\end{aligned}
$$

and $\xi_{1}=x-x_{0}, \xi_{2}=x+x_{1}$.

If, in contrast, $V_{1}$ has the form of a cylindrical capillary of radius $a$ and infinite length that is located parallel to the $z$ axis at a distance $x$ from the surface, we can write the solution of Eq. (1) as a superposition of the correlation function in the homogeneous case (incident wave in terms used for diffuse photon-density waves; see Refs. 8 and 9) given by Eq. (3) and the term that accounts for the presence of the cylindrical inhomogeneity (scattered wave). In the infinite medium for a plane-wave source described above, this important contribution depends only on the $y$ coordinate of the capillary axis ( $y=0$ corresponds to the centered capillary position with respect to the light detector) and, in our case of $D_{p}$ in $=D_{p}{ }^{\text {out }}$, is found to have the following form:

$$
\begin{aligned}
g_{1}{ }^{\text {scatt }}(y, \tau) \\
=-\frac{h-x_{0}}{2 \pi l^{*}} \sum_{n=1}^{\infty} \int_{-\theta_{1}}^{\theta_{1}} \frac{\mathrm{d} \theta_{s}}{\cos \theta_{s}} K_{n}\left(k_{\text {out }} \frac{h-x_{0}}{\cos \theta_{s}}\right) \\
\quad \times K_{n}\left(k_{\text {out }} \sqrt{h^{2}+y^{2}}\right) \cos \left[n\left(\theta_{s}-\theta\right)\right] \\
\quad \times\left[\frac{k_{\text {out }} I_{n}{ }^{\prime}\left(k_{\text {out }} a\right) I_{n}\left(k_{\text {in }} a\right)-k_{\text {in }} I_{n}\left(k_{\text {out }} a\right) I_{n}{ }^{\prime}\left(k_{\text {in }} a\right)}{k_{\text {out }} K_{n}{ }^{\prime}\left(k_{\text {out }} a\right) I_{n}\left(k_{\text {in }} a\right)-k_{\text {in }} K_{n}\left(k_{\text {out }} a\right) I_{n}{ }^{\prime}\left(k_{\text {in }} a\right)}\right] .
\end{aligned}
$$

Here $I_{n}$ and $K_{n}$ are modified Bessel functions, the prime indicates differentiation with respect to the argument, $h$ $=x+a$ denotes the $x$ position of the capillary axis, and $\theta=\arctan (y / h)$. $\theta_{1}$, which determines the limits of integration, is equal to $\pi / 2$ if an infinite plane-wave source is considered. A light source of finite extension in the direction of the $y$ axis can approximately be taken into account by use of a $\theta_{1}$ value that corresponds to the angular size of the source viewed from the capillary center.

To meet the null boundary condition on the plane $x$ $=-x_{1}$ and thus obtain $g_{1}{ }^{\text {scatt }}$ for the geometry of interest, we use the image method, i.e., we place the same capillary and a negative light source at the other side of this plane to obtain a symmetric geometry, as discussed in Refs. 8 and 13. Whereas Eq. (6) for the infinite medium is an exact solution of Eq. (1), the solution for a semiinfinite medium obtained with the image method is only approximate because only first-order scattered waves (in terms used for diffuse photon-density waves), are taken into account. In fact, the wave scattered from the actual capillary that is given by Eq. (6) hits the surface of the image capillary and gives rise to a second-order scattered wave, which in turn hits the capillary, and so on. Therefore an exact solution of the semi-infinite problem is an infinite series of consecutive scattered waves. Here we take only the first-order scattered waves, since they give the main contribution to the correlation function. Higher-order scattered waves cannot easily be taken into account but are negligible under most circumstances, as discussed in Ref. 8 . Note that both the above solutions for plane and cylindrical inclusions reduce to Eq. (3) for a homogeneous semi-infinite sample with pure Brownian motion if one assumes that $k_{\text {in }}(\tau)=k_{\text {out }}(\tau)$ for all $\tau$ or that $d=0$.

Although the solution for the cylindrical inhomogeneity [Eq. (6)] is much more complicated than the one for the planar inclusion [Eqs. (4) and (5)], the physical effects described by both of them are very similar. This is illustrated in Fig. 1, in which normalized time autocorrelation functions are plotted for the case in which the colloidal suspension is the same inside and outside the inclusion, flowing either in a cylindrical tube (solid curves) or in a planar sheet (dashed curves) for different depths $x$. In both cases there is no influence of the dynamical heterogeneity for $\tau=0$ (static experiment). All the curves are essentially identical at short times $\left(\tau \ll \tau_{f}^{2} / \tau_{0}{ }^{\text {in }}\right)$, where the overall decay is dominated by the (identical) Brownian motion inside and outside, because this decay is linear in $\tau$ as opposed to the $\tau^{2}$ dependence for flow. ${ }^{4}$ At intermediate correlation times the shear-flow-induced distortions are obviously largest for the shortest distances $x$. 


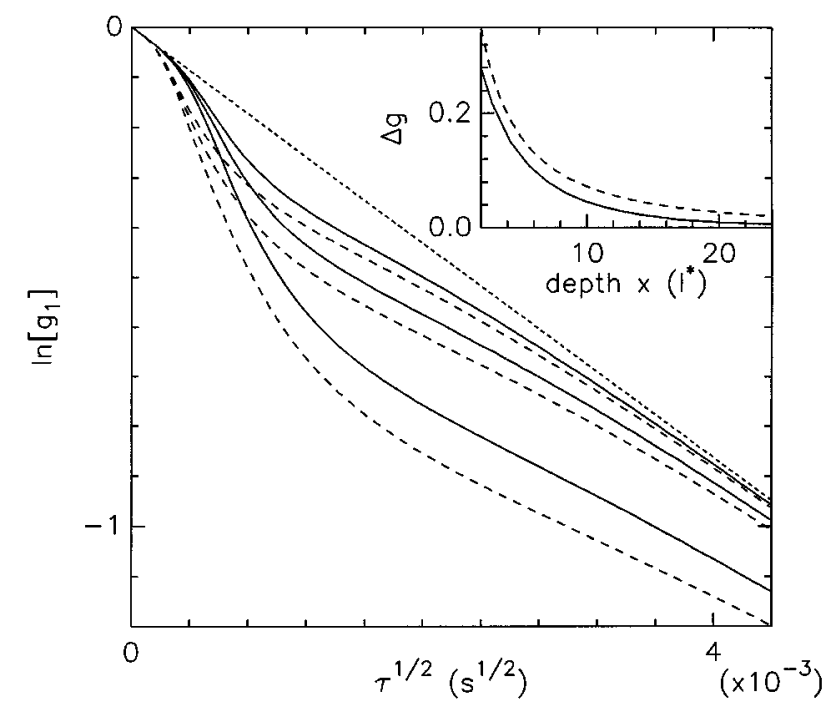

Fig. 1. Theoretical time correlation functions for cylindrical (solid curves) and planar (dashed curves) Poiseuille flows embedded in a semi-infinite homogeneous sample. The lower, middle, and upper pairs of curves correspond to depths $3 l^{*}, 5 l^{*}$, and $7 l^{*}$, respectively. $\quad y=0, d=22 l^{*}, \tau_{0}{ }^{\text {in }}=\tau_{0}^{\text {out }}=2.66 \times 10^{-4}$ $\mathrm{s}, \tau_{f}=4.6 \times 10^{-6} \mathrm{~s}$, and $\gamma=2.8$. The dotted line corresponds to a homogeneous semi-infinite medium. The inset shows the maximum absolute differences between the correlation functions with flow and with no flow (dotted line) as a function of flow $\operatorname{depth}(x)$.

In addition, at given $x$, they are larger for the case of planar inclusion, since in this case more photons scan regions of shear because of the infinite size of the sheet in the direction of the $y$ axis. At long correlation times all the curves tend to coincide with the curve for the homogeneous case (dotted curve). This can easily be explained ${ }^{10}$ by the time-path relation in dynamic multiple scattering, which associates long correlation times with short paths of diffusing photons, and vice versa. ${ }^{16}$ For long times $\tau$ the decrease of the correlation function is determined by short diffusion paths that do not reach sufficiently deep into the sample, on average, to hit the inclusion. A useful way to measure the influence of the inclusion is to calculate the maximum absolute value of the difference between the perturbed correlation function $g_{1}(\tau)$ and the unperturbed one, given by Eq. (3):

$$
\Delta g=\max _{0<\tau<\infty}\left|g_{1}(\tau)-g_{1}{ }^{0}(\tau)\right|,
$$

which is shown in the inset of Fig. 1 as a function of the inclusion depth $x$. It can be seen that significant perturbations of the correlation function due to flow inside the inclusion appear up to depths of approximately $10-15 l^{*}$ in this case.

\section{EXPERIMENT}

A vertically polarized beam of a monomode $\mathrm{Ar}^{+}$laser $(\lambda$ $=514.5 \mathrm{~nm}$; waist, $\approx 1 \mathrm{~nm})$ was incident upon the scattering sample cell (Fig. 2). The backscattered light was imaged from a 1-mm-diameter region of the cell surface through two pinholes and a lens onto a photomultiplier tube, thus collecting approximately one speckle spot. To reduce the influence of low-order scattering paths only depolarized light was detected ( $\mathrm{V}-\mathrm{H}$ configuration). The normalized intensity autocorrelation function $g_{2}(\tau)$ was calculated from the photomultiplier output with an electronic correlator. To cover the full time range of speckle fluctuations of interest, we used 88 correlator channels in multiple tau mode. We obtained the autocorrelation function of the scattered electric field $g_{1}(\tau)$ by applying the Siegert relation. ${ }^{19}$ Since the observation area of the scattered light necessarily had a nonvanishing size, the experimental values of $g_{1}(0)$ were always somewhat smaller than 1 , typically $0.75 \pm 0.01$. Hence, for quantitative comparison between experimental data and theory, the experimental $g_{1}(\tau)$ is multiplied by a constant.

The light-scattering cell was filled with a monodisperse suspension of polystyrene beads (particle diameter $b$ $=0.12 \mu \mathrm{m}, \tau_{0}{ }^{\text {out }}=2.66 \times 10^{-4} \mathrm{~s}, D_{B}{ }^{\text {out }}=3.55 \times 10^{-12}$ $\mathrm{m}^{2} / \mathrm{s}$, particle volume fraction $\Phi=0.058$, photon transport mean free path $\left.l^{*}=69 \mu \mathrm{m}\right)$. For visualization of flow, the capillary inside the cell contained this very same suspension, whereas for the case of a heterogeneity with distinct Brownian dynamics rather than flow the capillary was filled with a suspension of polystyrene beads of different particle diameters. This was realized with two different suspensions: $b=0.7 \mu \mathrm{m}$ and $b=2.04 \mu \mathrm{m}$, with $\tau_{0}{ }^{\text {in }}=1.55 \times 10^{-3} \mathrm{~s}\left(4.52 \times 10^{-3} \mathrm{~s}\right) ; D_{B}{ }^{\text {in }}=6.08$ $\times 10^{-13} \mathrm{~m}^{2} / \mathrm{s}\left(2.09 \times 10^{-13} \mathrm{~m}^{2} / \mathrm{s}\right) ; \Phi=0.035$ (0.067), respectively. The concentration of the suspension was always adjusted to match $l^{*}=69 \mu \mathrm{m}$ of the surrounding liquid, and the value $l^{*}=69 \mu \mathrm{m}$ was independently checked by standard multiple-light-scattering experiments in transmission geometry. ${ }^{16}$ By measuring the static backscattered intensity for different $y$ positions of the capillary, we carefully checked the absence of static scattering contrast both for the Brownian heterogeneities and for the glass wall of the capillary. To obtain an experimental $\gamma$ value [Eq. (3)], a reference measurement

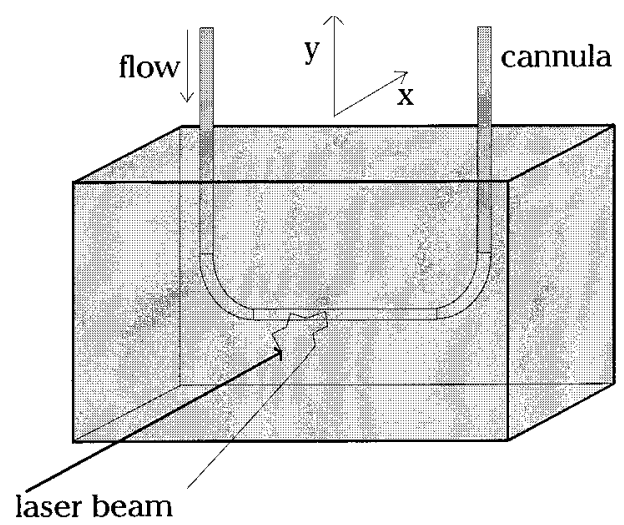

Fig. 2. Sketch of the light-scattering cell for realization of dynamic heterogeneities inside a turbid liquid. A large cell (5 $\mathrm{cm} \times 4 \mathrm{~cm} \times 2 \mathrm{~cm}$ ) is completely filled with a concentrated colloidal suspension. The included cylinder consists of an x-ray capillary (optical glass; length: $3 \mathrm{~cm}$, diameter $d=1.5 \mathrm{~mm}$, wall thickness: $0.01 \mathrm{~mm}$ ). A tube connected to the capillary delivers suspension from an elevated tank at flow rates controlled by its height. For the Brownian inclusions the capillary is filled with the corresponding suspensions. $x=0$ : sample surface, $y=0$ : center of the capillary. 


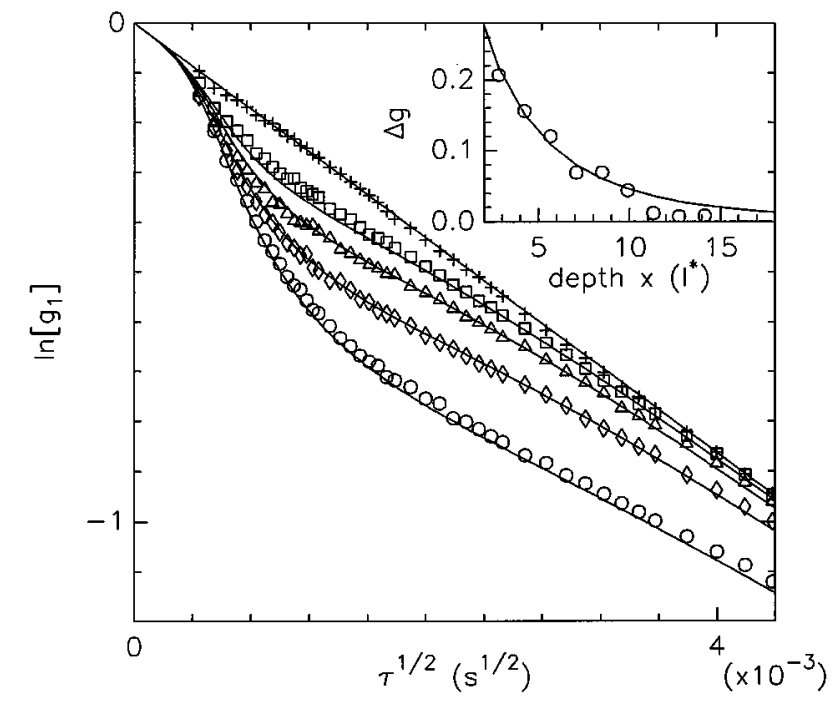

Fig. 3. Experimental time correlation functions for various distances $x$ of the capillary surface from the inner surface of the sample cell $\left[x=2.8 l^{*}(\bigcirc), 4.2 l^{*}(\diamond), 5.7 l^{*}(\triangle), 7.1 l^{*}(\square)\right.$; $y=0]$ and $Q=0.50 \mathrm{ml} / \mathrm{s}$ compared with the case of no flow (+). The inset shows the maximum difference $\Delta g$ of these correlation functions with respect to the Brownian case as a function of the $x$ position of the capillary. The theoretical predictions are indicated as solid curves.

without capillary was made for every dynamic inhomogeneity. For reasons of finite laser-beam size and because of a slightly different experimental setup for Brownian and flow measurements, ${ }^{20}$ these values range from 2.12 to 2.82 .

\section{RESULTS AND DISCUSSION}

\section{A. Visualization of Flow}

As shown in Fig. 1, the visualization of flow in a hidden slab or a capillary is possible within a finite window in correlation times. At sufficiently short times and sufficiently long times the decay of $g_{1}(\tau)$ is always dominated, at finite shear rates, by the Brownian motion. At short times this is due to the different time dependence of $g_{1}(\tau)$ for Brownian motion $\left(\sqrt{\tau / \tau_{0}{ }^{\text {in }}}\right)$ and shear motion $\left(\tau / \tau_{f}\right)$; at long times, because of the shortness of the contributing scattering paths that do not reach the heterogeneity buried at a finite depth. In Fig. 3 we compare the predicted function $g_{1}(\tau)$ for the case of the hidden capillary at different $x$ positions, together with the corresponding set of data. ${ }^{10}$ We find excellent agreement between data and theory over the entire range of available data. Note that all the relevant experimental parameters $\left(l^{*}, \gamma, d, x\right.$, $\tau_{0}{ }^{\text {in }}, Q$ ) were independently measured, and hence there is no adjustable fitting parameter. The remarkable agreement shown in Fig. 3 demonstrates the validity of the diffusion approach for the given typical set of parameters.

Closer inspection of the case without flow, shown in Fig. 3, reveals a small deviation of the data from the $\sqrt{\tau}$ behavior expected for plane-wave backscattering from a homogenous medium at short times. The effect is shown more clearly in Fig. 4, in which we compare backscattering data from the same sample, using two incident beam waists of fivefold different size. The $\sqrt{\tau}$ behavior is recovered for the larger beam waist. We therefore attribute the small short-time deviation in our typical data to the use of a smaller laser-beam size ( $1 \mathrm{~mm})$, which is necessary for visualizing millimeter-size objects. ${ }^{21}$ In Fig. 5 a set of data taken at fixed $x=2.8 l^{*}$ is shown for different flow rates. The major effect of increasing flow rate is to increase the time decay of $g_{1}(\tau)$ at intermediate $\tau$, that is, for $\tau>\tau_{f}^{2} / \tau_{0}$ in . The data shown are not inconsistent

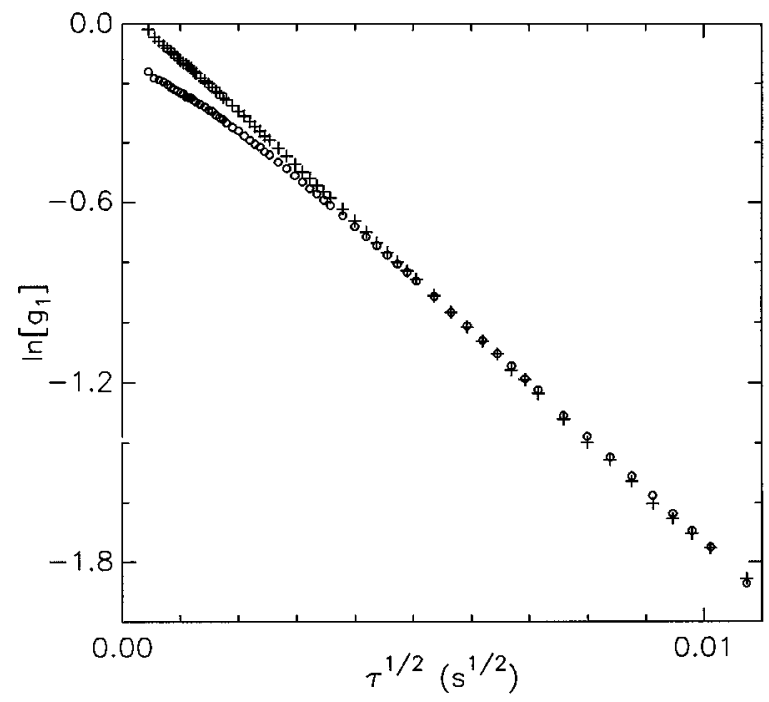

Fig. 4. Time correlation functions for backscattering from the suspension $\left(l^{*}=69 \mu \mathrm{m}, D=3.55 \times 10^{-12} \mathrm{~m}^{2} / \mathrm{s}\right)$, without included heterogeneity for different laser-beam sizes. The beam waist is roughly equal to $1 \mathrm{~mm}(\bigcirc)$; the expanded beam diameter is roughly equal to $5 \mathrm{~mm}(+)$.

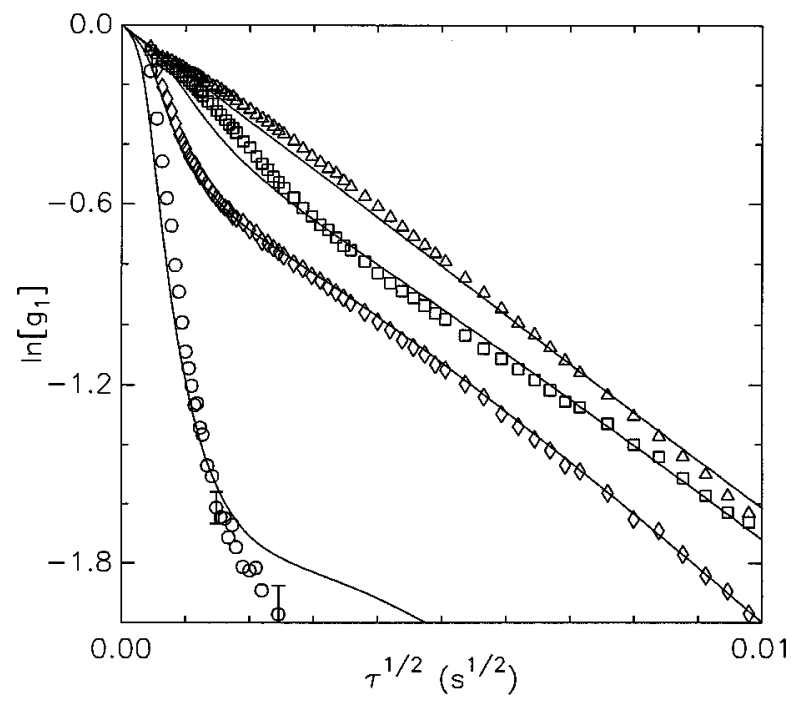

Fig. 5. Time correlation functions for different flow rates $Q$. The capillary is placed at $x=2.8 l^{*}$ inside the cell and is centered with respect to the incoming laser beam $[y=0 ; Q$ $=0.90 \mathrm{ml} / \mathrm{s}(\bigcirc), Q=0.50 \mathrm{ml} / \mathrm{s}(\diamond), Q=0.22 \mathrm{ml} / \mathrm{s}(\square)$, no flow $(\triangle)]$. The theoretical predictions are indicated as solid curves. 


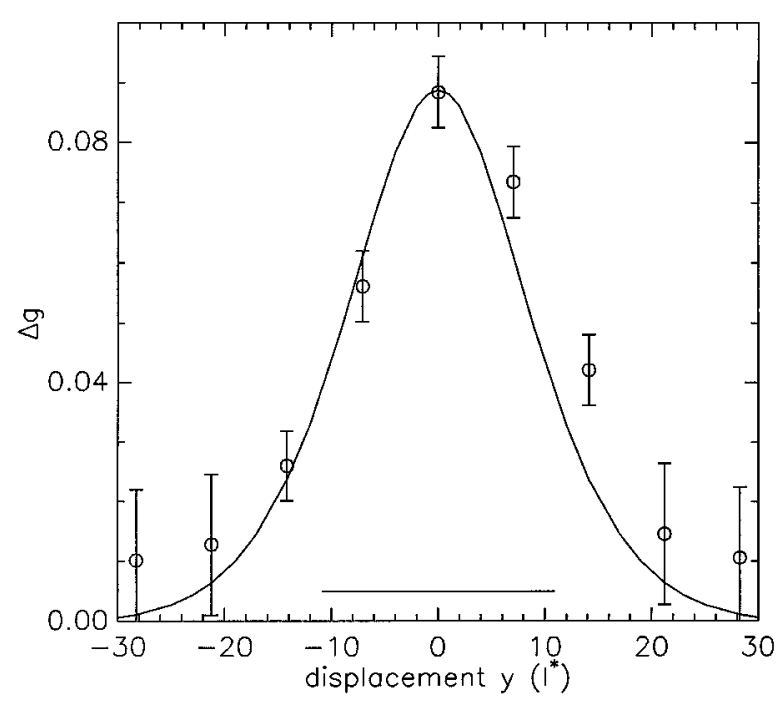

Fig. 6. For a fixed $x$ position of $7.1 l^{*}$ inside the cell, $\Delta g$ is shown as a function of the $y$ position of the flow $(Q=0.50 \mathrm{ml} / \mathrm{s})$. The error bars are due to uncertainty in determining $\Delta g$; the width of the capillary is indicated by the horizontal line. The solid curve denotes our theoretical prediction.

with the idea that the upper limit of the time window of the effect of flow depends not on the flow rate but merely on $x .^{10}$ The agreement of the data with the diffusion theory is very good, but slightly less than in Fig. 3 because of the small effect of the finite beam waist discussed above. In addition, the deviations seen at largest $\tau$ at the highest flow rate are due to small uncertainties in the measured average scattering intensity.

Finally, Fig. 6 shows measured and theoretical values of $\Delta g$ as a function of the $y$ position of the capillary for fixed $x$, fixed laser incidence, and fixed area of observation. Again, theory and data agree within the experimental errors without an adjustable parameter. The slight-and perhaps insignificant-asymmetry of data with respect to theory is attributed to the experimental difficulty of defining the center-of-gravity position and the diameter of the capillary to better than a few $l^{*}$.

For practical applications of the described method, it may appear mathematically rather involved to fit the experimental curves $g_{1}(\tau)$ individually, with $x, y, d, \tau_{0}^{\text {in }}$, and $Q$ as independent fitting parameters. Obviously, estimates of $y, \tau_{0}{ }^{\text {in }}$, and eventually $d$ can be obtained directly from a transverse scan in the $y$ direction, as shown in Fig. 6. The remaining parameters $x$ and $Q$ are easily obtained by a two-parameter fit, since the short-time slope of $g_{1}(\tau)$ is sensitive to $Q$ and $x$, whereas the longtime behavior of $g_{1}(\tau)$, that is, the time of approach of the case of no flow, depends only on $x$.

\section{B. Visualization of Distinct Brownian Motion}

So far, we have considered the case of flow versus Brownian motion of otherwise identical suspensions. However, the idea of visualizing dynamic heterogeneities is not restricted to different types of particles' motion, which give rise to different $\tau$ dependences of $g_{1}(\tau)$. Dynamic contrast can also be provided [without flow and, hence, with identical $\tau$ dependences of $g_{1}(\tau)$ ] by use of suspensions with different diffusion constants inside and outside the confined volume. This is illustrated in Fig. 7, in which we show the time dependence of $g_{1}(\tau)$ for the case of a capillary filled with larger colloidal particles in Brownian motion. As above, $l^{*}$ values are adjusted to be identical $\left(l^{*}=69 \mu \mathrm{m}\right)$ inside and outside the capillary. The observed dynamic contrast is now positive, since the larger

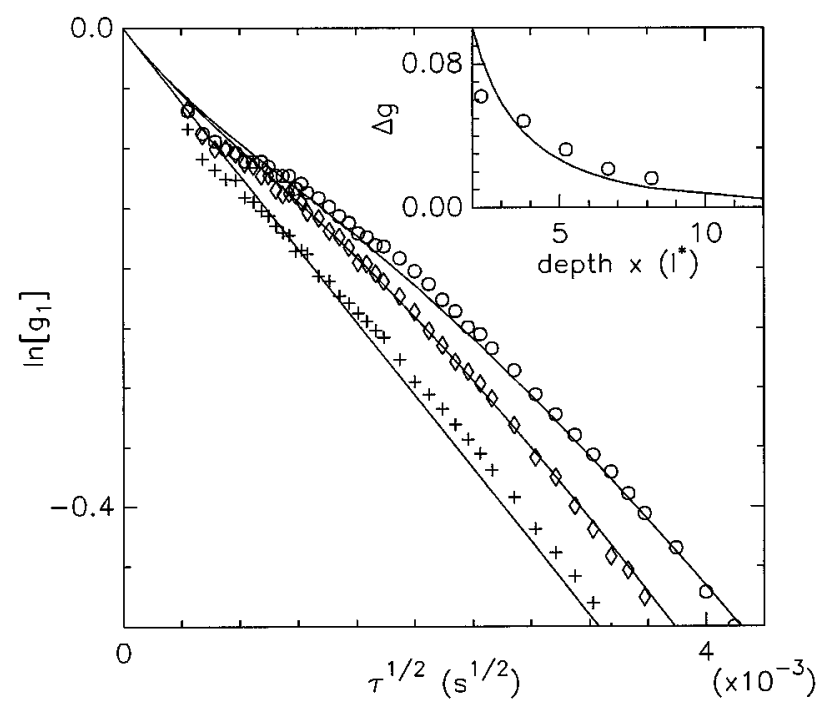

Fig. 7. Time correlation functions for different positions of the Brownian heterogeneity $\left[D_{B}{ }^{\text {in }}=2.09 \times 10^{-13} \mathrm{~m}^{2} / \mathrm{s} ; x=2.9 l^{*}\right.$ $\left.(\bigcirc), 5.22 l^{*}(\diamond) ; y=2.9 l^{*}\right]$ compared with the homogeneous case without inclusion ( + ). The inset shows $\Delta g$ of these correlation functions as a function of the $x$ position of the capillary. The theoretical predictions are indicated as solid curves.

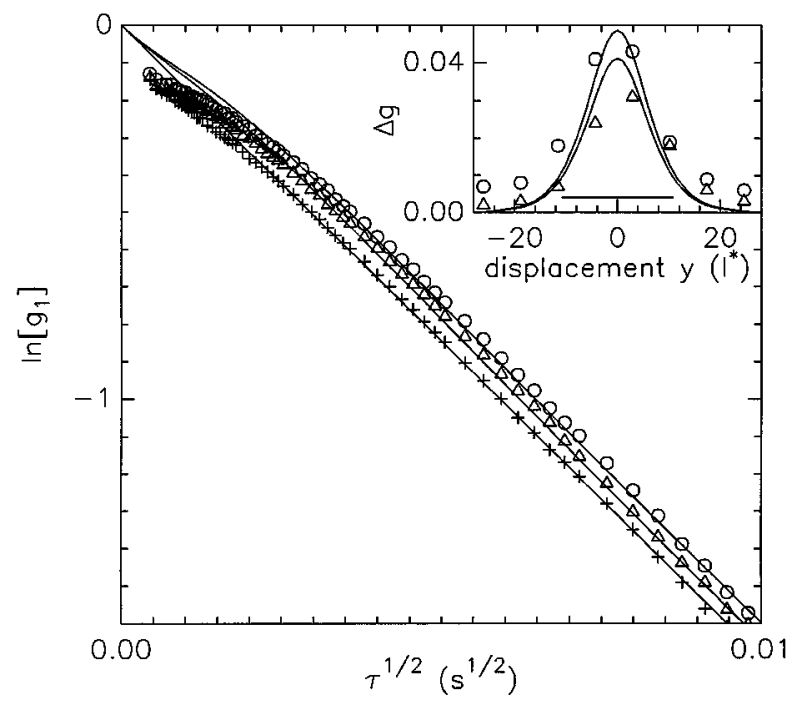

Fig. 8. Time correlation functions for different Brownian heterogeneities. The capillary is placed at $x=3.77 l^{*}, y=2.9 l^{*}$, inside the cell $\left[D_{B}{ }^{\text {in }}=2.09 \times 10^{-13} \mathrm{~m}^{2} / \mathrm{s} \quad(\bigcirc), D_{B}{ }^{\text {in }}=6.08\right.$ $\times 10^{-13} \mathrm{~m}^{2} / \mathrm{s}(\triangle), D_{B}{ }^{\text {in }}=D_{B}$ out $\left.=3.55 \times 10^{-12} \mathrm{~m}^{2} / \mathrm{s}(+)\right]$. Inset: For a fixed $x$ position of $3.77 l^{*}$ inside the cell, $\Delta g$ is shown as a function of the $y$ position for these heterogeneities. The solid curve denotes the theoretical prediction, and the width of the capillary is indicated by the horizontal line. 
particles in the tube slow down the decay of $g_{1}(\tau)$. Note that, unlike in the case of flow, the correlation functions now differ, in principle, at all small (but finite) $\tau$, since both contributions initially decay as $\sqrt{\tau}$. At long times, in contrast, all the curves are expected to merge into the curve for the homogeneous case at the same time as above. As discussed previously, this time is controlled only by $x$ and $\tau_{0}{ }^{\text {out }}{ }^{10}$ Figure 8 shows experimental and theoretical curves for two different particle sizes in comparison with the case of no contrast (the same particle size inside and outside). The inset gives the corresponding $y$ profiles. It appears that the effect due to Brownian contrast is generally smaller than the effect of flow and, in particular, that the two diffusion constants studied here are rather difficult to distinguish. Nevertheless, contrast is clearly visible over a very similar range of distances $x$ and is equally well described by our model.

\section{CONCLUSIONS}

In this paper we describe the physics of time-dependent multiple-scattering speckles generated by optical backscattering from a colloidal sample into which is inserted a thin capillary containing colloidal particles with different dynamical properties. The experimental and the theoretical material presented here illustrates that the method of visualizing dynamic heterogeneities by spaceand time-resolved measurements of the autocorrelation function of the multiple-scattered intensity works very well when the dynamic contrast is sufficiently large. Then, by comparison of data with simple diffusion theory, the method provides even quantitative information on the distance of the heterogeneity from the surface and on the type of motion occurring inside and outside. We can see with no particular effort a capillary of approximately $20 l^{*}$ diameter when it is positioned up to approximately $10-20 l *$ inside a liquid turbid suspension. This is similar to the typical performance in static incoherent multiple-scattering imaging. ${ }^{7}$ What remains to be seen is the ultimate limits of the method and how a true twodimensional image can be obtained with realistic technical effort and acceptable data-acquisition time.

\section{ACKNOWLEDGMENTS}

We kindly acknowledge fruitful discussions with $D$. Bicout and B. A. van Tiggelen. M. Heckmeier acknowledges the financial support provided by the Deutscher Akademischer Austauschdienst through the HSPII/ AUFE program.

*Also with Department of Physics, Moscow State University, 119899, Moscow, Russia.

\section{REFERENCES AND NOTES}

1. G. Maret and P. E. Wolf, "Multiple light scattering from disordered media. The effect of Brownian motion of scatterers," Z. Phys. B 65, 409-413 (1987).

2. D. A. Weitz, D. J. Pine, P. N. Pusey, and R. J. A. Tough, "Nondiffusive Brownian motion studied by diffusing-wave spectroscopy," Phys. Rev. Lett. 63, 1747-1750 (1989).

3. X. L. Wu, D. J. Pine, P. M. Chaikin, J. S. Huang, and D. A Weitz, "Diffusing-wave spectroscopy in a shear flow," J. Opt. Soc. Am. B 7, 15-20 (1990).

4. D. Bicout and G. Maret, "Multiple light scattering in Taylor-Couette flow," Physica A 210, 87-112 (1994).

5. W. Leutz and G. Maret, "Ultrasonic modulation of multiply scattered light," Physica B 204, 14-19 (1995).

6. D. J. Durian, D. A. Weitz, and D. J. Pine, "Multiple lightscattering probes of foam structure and dynamics," Science 252, 686-688 (1991).

7. P. N. den Outer, Th. M. Nieuwenhuizen, and A. Lagendijk, "Location of objects in multiple-scattering media," J. Opt. Soc. Am. A 10, 1209-1218 (1993).

8. D. A. Boas, M. A. O'Leary, B. Chance, and A. G. Yodh, "Scattering of diffuse photon density waves by spherical inhomogeneities within turbid media: analytic solution and applications," Proc. Natl. Acad. Sci. USA 91, 4887-4891 (1994).

9. D. A. Boas, L. E. Campbell, and A. G. Yodh, "Scattering and imaging with diffusing temporal field correlations," Phys. Rev. Lett. 75, 1855-1858 (1995).

10. M. Heckmeier and G. Maret, "Visualization of flow in multiple scattering liquids," Europhys. Lett. 34, 257-262 (1996).

11. P. M. Chaikin, D. J. Pine, D. A. Weitz, and E. Herbolzheimer, "Diffusing-wave spectroscopy," Phys. Rev. Lett. 60, 1134-1137 (1988).

12. A. Ishimaru, Wave Propagation and Scattering in Random Media (Academic, New York, 1978), pp. 175-190.

13. M. S. Patterson, B. Chance, and B. C. Wilson, "Time resolved reflectance and transmittance for the noninvasive measurement of tissue optical properties," Appl. Opt. 28, 2331-2336 (1989).

14. M. J. Stephen, "Temporal fluctuations in wave propagation in random media," Phys. Rev. B 37, 1-5 (1988).

15. D. Bicout, E. Akkermans, and R. Maynard, "Dynamical correlations for multiple light scattering in laminar flow," $\mathrm{J}$ Phys. (Paris) I 1, 471-491 (1991).

16. D. J. Pine, D. A. Weitz, G. Maret, P. E. Wolf, E. Herbolzheimer, and P. M. Chaikin, "Dynamical correlations of multiply scattered light," in Scattering and Localization of Classical Waves in Random Media, P. Sheng, ed. (World Scientific, London, 1989), pp. 312-372.

17. J. J. Duderstadt and L. J. Hamilton, Nuclear Reactor Analysis (Wiley, New York, 1976), pp. 140-144.

18. R. C. Haskell, L. V. Swaasand, T. Tsay, T. Feng, M. S. McAdams, and B. J. Tromberg, "Boundary conditions for the diffusion equation in radiative transfer," J. Opt. Soc. Am. A 11, 2727-2741 (1994).

19. B. J. Berne and R. Pecora, Dynamic Light Scattering (Wiley, New York, 1976), p. 40.

20. The laser beam is incident with an angle of approximately $10^{\circ}$ with respect to the backscattering direction. For different setups this angle changes slightly; hence the illuminated regions are not identical, and one obtains somewhat different $\gamma$ values.

21. However, we cannot exclude additional small deviations between data and theory that are due to neglect of higherorder scattering of the diffusing waves. 